\section{Ocular surface analysis in hematological patients before and after allogeneic hematopoietic stem cell transplantation: implication for daily clinical practice}

G Giannaccare ${ }^{1,3}$, F Bonifazi ${ }^{2,3}$, M Sessa², E Dan², M Arpinati², M Fresina', G Bandini², M Cavo², P Versura ${ }^{1}$ and EC Campos $^{1}$

\author{
Abstract \\ Purpose To evaluate ocular surface \\ parameters before and after hematopoietic \\ stem cell transplantation (HSCT) and to \\ correlate them with clinical and transplant \\ variables. \\ Methods This is a retrospective analysis of \\ data from 93 patients affected by \\ hematological malignancies undergoing \\ HSCT. Values from Ocular Surface Disease \\ Index, Schirmer test, Break-up Time, ocular \\ surface staining, and Meibomian Gland \\ Dysfunction score obtained before HSCT and \\ 3-6 months after were retrieved from charts. \\ Diagnosis and staging of dry eye (DE) disease \\ was performed according to Dry Eye \\ WorkShop criteria. Graft-versus-host-disease \\ (GVHD) was classified according to the NIH \\ criteria. Odds ratios for DE onset after HSCT \\ were estimated for demographic, ocular, \\ hematological and transplant variables. \\ Results DE was diagnosed before HSCT in \\ $50(53 \%)$ of the patients, mostly of \\ hyperevaporative profile. After HSCT, all \\ ocular parameters significantly worsened with \\ no change in DE profile. A $51 \%$ incident cases \\ (22 of the 43 non-DE subjects) were reported. \\ Increasing recipient age and female sex, \\ higher CD34+ cells infused, donor-recipient \\ sex mismatch (males receiving from females), \\ related donors, and peripheral blood cells as \\ stem cell source were associated with a \\ significant higher incidence of DE after \\ HSCT. Systemic chronic GVHD was \\ diagnosed in $42 \%$ while ocular GVHD \\ in $35.5 \%$ of the patients, which decreased \\ to $12 \%$ when taking into account only \\ incident cases.
}

Conclusions High DE prevalence was shown already before HSCT. A pre-HSCT ocular surface assessment is recommended for early DE diagnosis and treatment. This new protocol also influences the prevalence of ocular GVHD.

Eye (2017) 31, 1417-1426; doi:10.1038/eye.2017.78; published online 19 May 2017

\section{Introduction}

Graft-versus-host disease (GVHD) is the major complication of allogeneic hematopoietic stem cell transplantation (allo-HSCT) affecting both length and quality of life. ${ }^{1,2}$ Ocular GVHD develops in $30-60 \%$ of patients after allo-HSCT and in $60-90 \%$ of patients with acute or chronic systemic GVHD. ${ }^{3-5}$ It can affect all the structures of the eye from the cornea to the retina, reducing visual acuity and restricting daily activities. ${ }^{6}$ Dry eye (DE) disease is the hallmark of ocular chronic GVHD and is used for its diagnosis, scoring, and prognosis assessment. ${ }^{7-10}$ The National Institutes of Health (NIH) Criteria identified the new onset of dry, 'gritty', or painful eyes, cicatricial conjunctivitis, keratoconjunctivitis sicca, and confluent areas of punctate keratopathy as distinctive manifestations of chronic ocular GVHD. The updated 2015 NIH classification removed the Schirmer test, owing to its poor accuracy, from the severity scoring system that now includes frequency of lubricant eye drops usage and vision impairment. Ocular surface impairment represents a distinctive sign and therefore not considered to be sufficient to diagnose chronic GVHD if at least another organ is not involved. ${ }^{10}$
${ }^{1}$ Ophthalmology Unit, DIMES, Alma Mater Studiorum, University of Bologna, S. OrsolaMalpighi Teaching Hospital, Bologna, Italy

\section{${ }^{2}$ Haematology Institute 'L.A. Seragnoli', DIMES, Alma Mater Studiorum University of Bologna, S. Orsola-Malpighi Teaching Hospital, Bologna, Italy}

Correspondence: P Versura, Ophthalmology Unit, DIMES, University of Bologna, S.Orsola-Malpighi Hospital, Pad. 1 Palagi, Via Palagi, 9 Bologna 40138 Italy Tel: +39051 2142850; Fax: +39051342821 E-mail: piera.versura@ unibo.it

${ }^{3}$ These authors contributed equally and should be considered co-first authors.

Received: 21 April 2016 Accepted in revised form: 23 February 2017 Published online: 19 May 2017 
Several studies described ocular surface involvement in hematological patients after HSCT and risk factors for the development of ocular GVHD. ${ }^{5,11-15}$ Clinical findings include lacrimal gland dysfunction with keratoconjunctivitis sicca, conjunctival hyperemia and chemosis, pseudomembranous and cicatricial conjunctivitis, meibomian gland dysfunction, corneal epitheliopathy, filaments, painful erosion, and corneal ulceration. There are high concordance rates between acute and chronic systemic GVHD, and acute GVHD has been shown to be a strong predictor of chronic GVHD. ${ }^{16}$ Other major risk factors for ocular GVHD development include skin and mouth involvement, peripheral blood (PB) stem cell transplantation, and multiparous female donor to male recipient. ${ }^{5,11}$ Conversely, little information is available about ocular surface changes after HSCT compared with pretransplant baseline condition. ${ }^{17}$

A comprehensive baseline ophthalmological evaluation before HSCT has been recently recommended by the First International Chronic Ocular GVHD Consensus Group, the German-Austrian-Swiss Consensus Conference, and the 2015 updated NIH Consensus Conference, with the aim to classify the onset of ocular symptoms and signs after HSCT as incident cases. ${ }^{10,18,19}$ On the contrary, if DE is already present before HSCT, current criteria to diagnose ocular GVHD may be not fulfilled.

The purpose of the present study was to comprehensively evaluate ocular surface parameters in the same hematological patients before and after allogeneic transplantation and to correlate them with clinical and transplant variables.

\section{Materials and methods}

\section{Patients and transplant procedure}

Data had been collected over the period March 2007 to March 2014. The study followed the tenets of the Declaration of Helsinki and was approved by the ethics committee of the S.Orsola-Malpighi Teaching Hospital. Informed consent was obtained from all patients when returning for subsequent check-ups. In our practice, the two scheduled ophthalmological visits are performed before HSCT and conditioning regimen (V0) and in a time window ranging from 3 to 6 months after HSCT (V1). ${ }^{20}$ Data from 203 patients undergoing HSCT at the Hematology Institute "L.A. Seragnoli", University of Bologna, S. Orsola-Malpighi Teaching Hospital in Bologna (Italy) were retrieved. Only the 113 charts containing ophthalmological data to be included in the study analysis and collected at V0 and V1 were further selected. Cases excluded referred to: 28 patients who had not received an ophthalmological visit in the first 6 months after HSCT owing to poor general health conditions or for the occurred death; 25 patients whose charts had not been found to be fully completed; and 37 patients living out of our area who had been referred to another eye Center in the post-HSCT follow-up. Twenty further cases were excluded for the history of uveitis $(n=4)$, retinitis $(n=8)$, and concomitant use of eye drops for the treatment of glaucoma $(n=8)$.

Ninety-three Caucasian patients fulfilled the criteria and were then finally included in the study. For the statistical analysis, patients were divided into two groups according to the underlying disease: chronic lymphoproliferative disorders, including Hodgkin lymphoma, non-Hodgkin lymphoma, multiple myeloma, chronic lymphocytic leukemia, and stem cell malignancies, including acute myeloid leukemia (AML), acute lymphoblastic leukemia (ALL), myelodysplastic syndrome, and chronic myeloid leukemia (CML). The disease phase at transplant was classified as early and advanced. Patients with CML in first chronic phase, ALL and AML in the first complete remission and lymphomas in complete remission were considered as early phase; all the remaining cases were considered as advanced phase. Twenty patients $(21.5 \%$ of the total) received HSCT from human leukocyte antigen (HLA)-identical siblings (related donors (RDs)) and $73(78.5 \%)$ from voluntary unrelated donors. HLA matched were defined all the pairs 10/10 loci matched; if the matching was $<10 / 10$ they were classified as mismatch. Sources of hematopoietic stem cells were bone marrow (BM) in 41 cases $(44.0 \%), \mathrm{PB}$ in 46 cases $(49.5 \%)$, and cord blood (CB) in 6 cases $(6.5 \%)$. In the analysis, stem cells from BM and $\mathrm{CB}$ were considered in the same group compared with $\mathrm{PB}$. The intensity of conditioning regimen was standard in 64 patients $(69 \%$ of the total) and reduced intensity in 29 patients $(31 \%)$. Conditioning regimens were busulfanbased (54 patients) or total body irradiation (unfractioned, 800 cGy from Linear Accelerator at low dose rate) based (10 patients). Reduced intensity regimens were Tiothepa $10 \mathrm{mg} / \mathrm{kg}$-cyclophosphamide $60 \mathrm{mg} / \mathrm{kg}$-fludarabine $60 \mathrm{mg} / \mathrm{sm}$ (20 patients) or Melphalan-Fludarabine (9 patients). All patients received GVHD prophylaxis with Cyclosporin-A and short-term Methotrexate (days 1, 3, 6, 11 with 15, 10, 10, $10 \mathrm{mg} / \mathrm{sm}$, respectively) or Mycophenolate mofetil ( $15 \mathrm{mg} / \mathrm{kg}$ bid from day +1 to day +30 ). In addition, all transplants from unrelated donors received antylymphocyte globulin (ATG-F, Grafalon, Bad Homburg, Germany) at $15-30 \mathrm{mg} / \mathrm{kg}$ total dose while only $11(55 \%)$ patients receiving transplants from their HLA-identical sibling did. Ocular and systemic GVHD were classified by the standard NIH criteria, that is, the ocular involvement is only a distinctive manifestation and an additional distinctive feature of another organ is necessary to establish diagnosis., 70 Patients were allocated in single, air-positive pressure rooms with 
HEPA-filtered air. Anti-infectious prophylaxis was accomplished with levoxacin and fluconazole during the transplant period and acyclovir and cotrimoxazole until the ninth month after transplant. In case of cytomegalovirus (CMV) DNA positivity, CMV preemptive therapy was administered using gancyclovir or foscarnet if gancyclovir was contraindicated. All patients received filtered and irradiated blood products.

\section{Ophthalmological examination}

Ophthalmological examinations were always performed before HSCT and conditioning regimen (V0) and 3-6 months later (V1), as described elsewhere. ${ }^{20}$ Briefly, subjective discomfort symptoms were graded 0-100 with the Ocular Surface Disease Index (OSDI) score. ${ }^{21}$ The DE examination was performed from the least to the most invasive test as follows: tear stability was measured by tear film break up time (TFBUT; average of three measurements) using $2 \mu \mathrm{l}$ unpreserved $2 \%$ sodium fluorescein (galenic preparation from Fluoralfa, Alfa Intes, Italy); corneal and conjunctival fluorescein stainings were assessed under cobalt blue illumination with the aid of a 7503 Boston Yellow Filter Kit (equivalent to Kodak Wratten 12) to enhance staining details and graded according to the NEI (National Eye Institute) score and van Bijsterveldt score, respectively; ${ }^{22,23}$ tear production was estimated by the 5-min Schirmer test performed with sterile strips without anesthetic (ContaCare Ophthalmics and Diagnostics, Gujarat, India).

Conjunctival injection was graded as previously described $^{24}$ as stage I: hyperemia; stage II: hyperemia with serosanguinous chemosis; stage III:

pseudomembranous conjunctivitis; and stage IV: membranous/pseudomembranous conjunctivitis plus corneal epithelial sloughing.

Meibomian gland dysfunction (MGD) was assessed to grade the quality, expressibility, and volume of gland secretion, according to the modified MGD Faulks\&Bron scoring system (range 0-27). ${ }^{25}$

Classification of DE patients was based on a series of thresholds according to the Dry Eye WorkShop severity score $^{26}$ (DEWS, ranging from the less severe grade 1 to the most severe grade 4) and modified after Sullivan et al. ${ }^{27}$ The criteria required evidence of symptoms, with an OSDI score $>5$, and in addition, at least one eye had to exceed thresholds on two of the five subset signs, chosen from TFBUT $<8$, Schirmer test $\leq 7$, corneal staining $>0$, conjunctival staining $>0$, and MGD score $>5$ (Table 1 ). The DE severity was assigned depending on the highest number of values falling under each grade. Post- vs preHSCT changes were defined as worsening if the increase of at least one point in the level grade occurred.
Table 1 Dry eye (DE) staging according to the DE severity level proposed by the report of the Diagnostic Methodology Subcommittee of the International Dry Eye Workshop and modified according to Sullivan et al ${ }^{27}$

\begin{tabular}{lcccc}
\hline Dry eye severity grade & 1 & 2 & 3 & 4 \\
\hline OSDI score & $5-15$ & $16-30$ & $31-45$ & $46-100$ \\
TFBUT (s) & $7-10$ & $5-6$ & $2-4$ & $0-1$ \\
Corneal staining (NEI score) & $<3$ & $3-8$ & $9-12$ & $13-15$ \\
Conjunctival staining score & $<4$ & $5-8$ & $9-12$ & $13-18$ \\
Schirmer score (mm/5 min) & $7-10$ & $5-6$ & $2-4$ & $0-1$ \\
MGD score & $<5$ & $5-12$ & $13-20$ & $21-27$ \\
\hline
\end{tabular}

Abbreviations: MGD, Meibomian gland dysfunction; NEI, National Eye Institute; OSDI, Ocular Surface Disease Index; TFBUT (s), tear film break up time (seconds).

\section{Ocular treatment}

Ocular treatment was prescribed to patients affected by DE already at baseline according to DEWS guidelines driven by DE severity score. Briefly, hyaluronic-based tear substitutes, nocturnal ointment, and lid hygiene were prescribed in mild-moderate cases (DEWS severity grade 1-2) while additional anti-inflammatory therapy (steroids -loteprednol etabonate $0.5 \%$ ophthalmic suspension (Lotemax, Bausch and Lomb, Rochester, NY, USA, 4 times/day for 4 weeks ${ }^{28}$ ) or cyclosporine-galenic preparation $0.1 \%$ in oil, 2 times $/$ day $^{29}$ ) was prescribed in most severe cases (DEWS severity grade $3-4) .{ }^{30}$ The same rationale was used in patients developing or worsening DE condition after HSCT.

\section{Statistical analysis}

Statistical evaluation was performed by using the MedCalc statistical software 14.8 (Ostend, Belgium) and IBM SPSS statistics for windows, version 20.0. (Armonk, NY, USA) Data from both eyes were collected from charts, but only the value from the worst eye was taken into consideration for statistical purposes. All data were expressed as mean \pm SD and median (min value; max value) (95\% confidence interval for the median).

Pre- and post-HSCT values changes were evaluated by Wilcoxon test for related samples and Mann-Whitney test for independent samples. For each ocular parameter, the post- vs pre-HSCT values and the differences between post- and pre-HSCT values $(\Delta)$ were correlated to preHSCT values by Spearman coefficient correlation (small correlation strength 0.10-0.29; medium 0.30-0.49; large 0.50-1.00). Univariate (Chi-square test, odds ratio (OR)) and multivariate logistic forward regression analysis were used to assess the association between demographic, ocular, hematological, and transplant-related variables and DE after HSCT. Risk for DE was also estimated by OR with $95 \%$ confidence intervals that independently 
associated with the disease. Data were considered to be statistically significant if $P<0.05$.

\section{Results}

Demographic, hematological, and ophthalmological data were summarized in Table 2. The interval between HSCT and V1 visit was shown to be 115 days (97-150) (95-176) (median, 95\% CI, min-max value). Patients showed a similar distribution between genders, with a not statistically significant difference between males and female ages. Stratification by gender is of importance because the prevalence of DE is significantly higher in females. $^{31}$

\section{Pre-HSCT ophthalmological parameter analysis}

Forty-three patients (47\%) were classified as non-DE subjects, whereas 50 patients (53\%) were classified as DE sufferers. Of these, 25 patients were classified as DEWS modified score 1 ( $50 \%$ of the total DE-subjects), 21 patients as score 2 (42\% of the total), and 4 patients as score 3 ( $8 \%$ of the total) (Figure 1). Results of ophthalmological parameters for both non-DE and DE patients are summarized in the left column of Table 3A and $\mathrm{B}$, respectively. The DE patients appeared to be moderately symptomatic according to OSDI, with a normal tear production as median and a hyperevaporative DE type with tear instability and pathological MGD scores.

Hyaluronic-based tear substitutes, nocturnal ointment, and lid hygiene were prescribed to 46 patients (DEWS severity levels 1-2) while loteprednol etabonate $0.5 \%$ ophthalmic suspension was prescribed to the four most severe patients (DEWS severity level 3).

\section{Post-HSCT parameter analysis}

DE disease was present at the V1 visit in 72 patients $(77 \%$ of the total): 31 patients (33\%) were classified as DEWS severity level 1, 32 patients (34.5\%) as DEWS level 2, and 7 patients (9.5\%) as DEWS level 3 (Figure 1).

After HSCT, a statistically significant worsening of ocular parameters compared with pretransplant values was shown for both groups. Only conjunctival surface damage did not change in DE patients before HSCT, remaining comparable to values before transplant. Despite the significant reduction, the Schirmer test as a median after HSCT resulted in the normal range, whereas the TFBUT as a median was found in the pathological range.

Among the post-HSCT DE patients, 22 out of the 43 non-DE pre-HSCT subjects developed DE after: these incident cases showed different DEWS levels of severity
Table 2 Clinical and demographic variables in subjects included in the study

\begin{tabular}{|c|c|c|}
\hline & Patient number & $\begin{array}{l}\% \text { vs } \\
\text { total }\end{array}$ \\
\hline \multicolumn{3}{|l|}{ Demographic data } \\
\hline Females & 48 & 51.5 \\
\hline Males & 45 & 48.5 \\
\hline Age (years) & $46(18-64)(43-48)$ & \\
\hline \multicolumn{3}{|l|}{ Hematological history } \\
\hline \multicolumn{3}{|l|}{ Disorders } \\
\hline AML & 28 & 30.0 \\
\hline ALL & 19 & 20.5 \\
\hline HL & 9 & 9.5 \\
\hline CML & 9 & 9.5 \\
\hline NHL & 9 & 9.5 \\
\hline MM & 8 & 8.5 \\
\hline MDS & 7 & 7.5 \\
\hline CLL & 4 & 5.0 \\
\hline $\begin{array}{l}\text { Time from diagnosis (days) to } \\
\text { transplant to HSCT (days) }\end{array}$ & $\begin{array}{l}281(113-2783) \\
(231-464)\end{array}$ & \\
\hline \multicolumn{3}{|l|}{ Disease stage } \\
\hline Early & 34 & 36.5 \\
\hline Advanced & 59 & 63.5 \\
\hline Previous autograft & 9 & 9.6 \\
\hline \multicolumn{3}{|c|}{ Previous chemotherapy medications (no. of cycles) } \\
\hline$\leq 3$ cycles & 40 & 43.0 \\
\hline$>3$ cycles & 53 & 57.0 \\
\hline \multicolumn{3}{|l|}{ Ocular history } \\
\hline Contact lens wearers & 17 & 18.5 \\
\hline VDT users & $12^{\mathrm{a}}$ & 13.0 \\
\hline Previous ocular surgery & 7 & 7.5 \\
\hline \multicolumn{3}{|l|}{ HSCT parameter } \\
\hline \multicolumn{3}{|l|}{ Donor characteristics } \\
\hline Age (years) & $32(19-68)(28-33)$ & \\
\hline VUD & 73 & 78.5 \\
\hline HLA match & 26 & 28 \\
\hline HLA mismatch & 47 & 50.5 \\
\hline $\mathrm{RD}$ & 20 & 21.5 \\
\hline Sex mismatch & 45 & 48 \\
\hline \multicolumn{3}{|l|}{ Conditioning regimen } \\
\hline Reduced & 29 & 31 \\
\hline Myeloablative & 64 & 69 \\
\hline \multicolumn{3}{|l|}{ Stem cell source } \\
\hline Bone marrow & 41 & 44 \\
\hline Peripheral blood & 46 & 49.5 \\
\hline Cord blood & 6 & 6.5 \\
\hline
\end{tabular}

Abbreviations: ALL, acute lymphoblastic leukemia; AML, acute myeloid leukemia; CLL, chronic lymphocytic leukemia; CML, chronic myeloid leukemia; HL, Hodgkin lymphoma; MDS, myelodysplastic syndrome; MM, multiple myeloma; NHL, non-Hodgkin lymphoma; RD, related donors; VUD, voluntary unrelated donors. Data are expressed as median (min-max values) $(95 \% \mathrm{CI}) .{ }^{\text {a }} \mathrm{VDT}$, video terminal users defined as a worker who carries on business usually using equipment powered by a display screen, including laptops, for at least $20 \mathrm{~h}$ a week, i.e. for $>4 \mathrm{~h} /$ working day.

as shown in Figure 1. Also, these DE patients could be classified as hyper-evaporative DE type (mean values \pm SD: Schirmer test $20.4 \pm 12.5 \mathrm{~mm} / 5 \mathrm{~min}$; TFBUT $6.3 \pm 2.1 \mathrm{~s}$; MGD score $7.5 \pm 4.5)$. Results of 


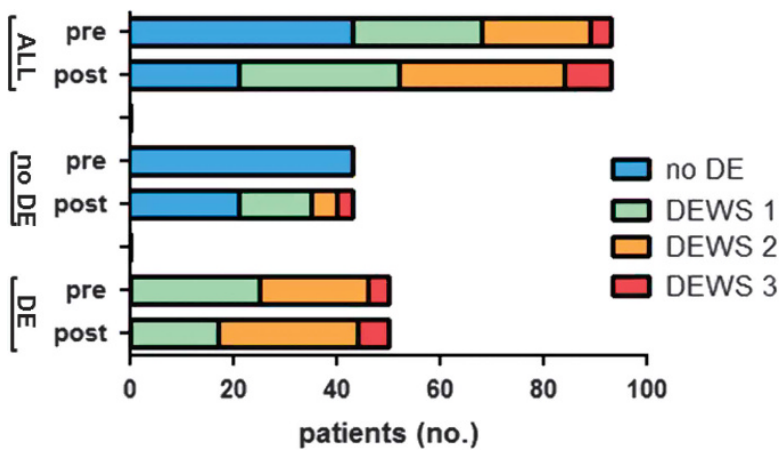

Figure 1 Distribution of dry eye (DE) disease staged according to modified severity level DEWS grade system ${ }^{27}$ in patients before and after HSCT. The distribution was shown for the whole population (ALL) in the upper part, for patients who had not been diagnosed as DE pre-HSCT (no-DE) in the middle part, and for patients who had been diagnosed as DE pre-HSCT (DE) in the lower part. ophthalmological parameters for non-DE and DE patients are summarized in the right column of Table $3 \mathrm{~A}$ and $\mathrm{B}$, respectively. Post-HSCT ophthalmological parameters from these two groups of patients were compared; a statistically significant difference was found with worst values shown in the DE pre-HSCT group of patients (always $P<0.001$ ).

Thirty-four out of the 50 DE pre-HSCT patients did not change severity grade, whereas the remaining 16 worsened their pre-HSCT DEWS severity level, mostly shifting from level 1 to level 2. The DE profile did not change after the transplant from hyperevaporative to aqueous deficiency or vice versa. Hyaluronic-based tear substitutes, nocturnal ointment, and lid hygiene were prescribed to 65 patients (DEWS severity levels 1-2) while loteprednol etabonate $0.5 \%$ ophthalmic suspension was

Table 3 Results from ophthalmological examinations in DE patients before and after HSCT

\begin{tabular}{|c|c|c|c|}
\hline Parameters & Pre-HSCT & Post-HSCT & $\mathrm{P}$ \\
\hline \multicolumn{4}{|c|}{ (A) Results for the group of subjects not diagnosed as suffering from DE before HSCT } \\
\hline OSDI score & $\begin{array}{c}6.4 \pm 7.4 \\
4(0-15)(2-6)\end{array}$ & $\begin{array}{c}15.2 \pm 14.7 \\
13(2-56)(7-19)\end{array}$ & $<0.0001$ \\
\hline TFBUT (s) & $\begin{array}{c}13.1 \pm 3.0 \\
15(4-15)(12-15)\end{array}$ & $\begin{array}{c}9.2 \pm 4.6 \\
8(1-15)(8-12)\end{array}$ & $<0.0001$ \\
\hline Schirmer test (mm length/5 min) & $\begin{array}{c}30.1 \pm 12.1 \\
40(10-40)(30-40)\end{array}$ & $\begin{array}{c}23.3 \pm 12.2 \\
20(2-40)(16-27)\end{array}$ & $<0.001$ \\
\hline Conjunctival staining (van Bijsterveldt score) & $\begin{array}{c}1.15 \pm 1.74 \\
0(0-5)(0-1)\end{array}$ & $\begin{array}{c}2.21 \pm 2.23 \\
2(0-8)(1-2)\end{array}$ & $<0.01$ \\
\hline Corneal damage (NEI score) & $\begin{array}{c}0 \pm 0 \\
0(0-0)(0-0)\end{array}$ & $\begin{array}{c}1.7 \pm 2.1 \\
0(0-6)(0-3)\end{array}$ & $<0.0001$ \\
\hline MGD score & $\begin{array}{c}1.5 \pm 1.1 \\
2.5(1-4)(2-3)\end{array}$ & $\begin{array}{c}6.5 \pm 5.5 \\
7.5(4-14)(7-12)\end{array}$ & $<0.0001$ \\
\hline Conjunctival injection & $\begin{array}{c}0.2 \pm 0.2 \\
0.4(0-1)(0-1)\end{array}$ & $\begin{array}{c}0.7 \pm 0.8 \\
0.8(0-2)(0-2)\end{array}$ & $<0.01$ \\
\hline \multicolumn{4}{|c|}{ (B) Results for the group of patients diagnosed as suffering from DE before HSCT } \\
\hline OSDI score & $\begin{array}{c}9.7 \pm 11.7 \\
6(6-59)(6-8)\end{array}$ & $\begin{array}{c}18.2 \pm 17.1 \\
15(6-73)(12-20)\end{array}$ & $<0.0001$ \\
\hline TFBUT (s) & $\begin{array}{c}6.8 \pm 2.3 \\
7.5(1-15)(7-8)\end{array}$ & $\begin{array}{c}6.0 \pm 2.1 \\
6.1(1-12)(5-7)\end{array}$ & $<0.01$ \\
\hline Schirmer test (mm length/5 min) & $\begin{array}{c}17.5 \pm 13.2 \\
15(9-40)(10-20)\end{array}$ & $\begin{array}{c}15.2 \pm 11.2 \\
10(1-25)(0-12)\end{array}$ & $<0.01$ \\
\hline Conjunctival staining (van Bijsterveldt score) & $\begin{array}{c}2.1 \pm 2.5 \\
3(0-9)(0-3)\end{array}$ & $\begin{array}{l}3.6 \pm 3.1 \\
3(0-9)(0-3)\end{array}$ & 0.4 \\
\hline Corneal damage (NEI staining score ) & $\begin{array}{c}1.9 \pm 2.0 \\
2(0-12)(1-2)\end{array}$ & $\begin{array}{c}2.1 \pm 2.4 \\
2(0-12)(2-4)\end{array}$ & $<0.01$ \\
\hline MGD score & $\begin{array}{c}6.5 \pm 3.5 \\
7(3-10)(5-8)\end{array}$ & $\begin{array}{c}9.1 \pm 7.5 \\
9.5(5-16)(8-13)\end{array}$ & $<0.01$ \\
\hline Conjunctival injection & $\begin{array}{c}0.5 \pm 0.5 \\
0.6(0-1)(0-1)\end{array}$ & $\begin{array}{c}0.9 \pm 0.8 \\
0.9(0-2)(0-2)\end{array}$ & $<0.01$ \\
\hline
\end{tabular}

Abbreviations: MGD, Meibomian gland dysfunction; NEI, National Eye Institute; OSDI, Ocular Surface Disease Index; TFBUT, tear film break up time. Values are expressed as mean \pm SD and median (minimum value- maximum value) ( $95 \%$ confidence interval). 
prescribed to the 7 most severe patients (DEWS severity level 3).

Systemic chronic GVHD was diagnosed in 39 patients ( $42 \%$ of the total) while ocular GVHD in 33 patients (35.5\% of the total) regardless of the presence of DE pre-HSCT, according to NIH Criteria.7,10 Conversely, the cases of ocular GVHD decreased to 11 cases $(12 \%)$ in our population when taking into account only incident cases. Also ocular GVHD patients could be classified as hyperevaporative DE type.

\section{Correlations}

A statistically significant correlation between posttransplant vs pretransplant values was only found for Schirmer test $(r h o=0.512)$ and OSDI score $($ rho $=0.461)$. The difference between pre- and post-HSCT values $(\Delta)$ was calculated and correlated to the corresponding preHSCT value. $\Delta$ values of all ocular parameters showed a significant inverse correlation with pre-HSCT values: TFBUT (rho $=-0.577$ ), Schirmer test (rho $=-0.605)$, OSDI $($ rho $=-0.364)$, NEI score $(r h o=-0.525)$, and van Bijsterveldt score (rho $=-0.534$ ) (always $P<0.001)$. This finding indicates a greater worsening after HSCT in patients who had already shown impaired parameters before HSCT.

\section{Univariate analysis}

In univariate analysis, increasing recipient age, recipient female sex, and higher CD34+ cells infused were associated with a significant higher incidence of DE after HSCT in those subjects not suffering from DE before HSCT (Table 4). Advanced disease stage at the time of HSCT, donor-recipient sex mismatch (males receiving from females), RDs, and PB cells as stem cell source were associated with a significant higher incidence of DE after HSCT in subjects either having or not having DE before HSCT (Tables 4 and 5).

\section{Multivariate analysis}

Multiple analysis of variables significant in the univariate, or clinically relevant, did not show any statistically significant $P$-value in subjects either having or not having DE before HSCT.

\section{Discussion}

In this retrospective study data from comprehensive ocular surface evaluation in the same patients before and after allo-HSCT were analyzed. To our knowledge, only another study was performed on 53 patients analyzing prospectively the same population before and after
Table 4 Univariate odds ratios (OR) and 95\% confidence intervals of associated factors in the subgroup of patients, defined as those not suffering from DE before HSCT, to develop DE after HSCT

\begin{tabular}{|c|c|c|}
\hline Variable & $\begin{array}{l}\text { Unadjusted OR } \\
\quad(95 \% \mathrm{CI})\end{array}$ & $P$ \\
\hline Recipient age (years) ${ }^{a}$ & $1.04(1.01-1.08)$ & 0.03 \\
\hline Recipient gender (female vs male) ${ }^{\mathrm{a}}$ & $1.43(1.20-4.43)$ & 0.01 \\
\hline \multicolumn{3}{|l|}{ Ocular variables } \\
\hline VDT use & $0.27(0.10-1.11)$ & 0.21 \\
\hline Previous eye surgery & $0.21(0.48-1.23)$ & 0.32 \\
\hline \multicolumn{3}{|l|}{ Hematological variables } \\
\hline Type of disease & $0.57(0.16-2.06)$ & 0.39 \\
\hline $\begin{array}{l}\text { Time from diagnosis to HSCT } \\
\text { (>6 months) }\end{array}$ & $0.81(0.28-2.34)$ & 0.71 \\
\hline Advanced disease stage $\mathrm{e}^{\mathrm{a}}$ & $1.23(1.08-3.55)$ & 0.02 \\
\hline $\begin{array}{l}\text { Previous chemotherapy } \\
\text { medications (no. of cycles) }\end{array}$ & $1.06(0.87-1.30)$ & 0.50 \\
\hline \multicolumn{3}{|l|}{ Transplant variables } \\
\hline Donor-recipent sex mismatch ${ }^{\mathrm{a}}$ & $1.33(1.12-4.47)$ & 0.03 \\
\hline Donor age & $1.07(0.98-1.16)$ & 0.07 \\
\hline Donor type (related donors) ${ }^{\mathrm{a}}$ & $7.50(1.56-35.9)$ & 0.03 \\
\hline Stem cell source $\left(\mathrm{PB}\right.$ cells) ${ }^{\mathrm{a}}$ & $2.12(1.49-5.02)$ & 0.01 \\
\hline Intensity of conditioning regimen & $0.71(0.29-1.74)$ & 0.45 \\
\hline HLA mismatch & $0.43(0.10-1.76)$ & 0.24 \\
\hline CD34+ cells infused ${ }^{\mathrm{a}}$ & $1.11(1.09-1.92)$ & 0.01 \\
\hline GVHD systemic ${ }^{\mathrm{b}}$ & $1.23(1.13-5.38)$ & 0.01 \\
\hline
\end{tabular}

Multiple analysis of variables significant in the univariate or clinically relevant did not show any statistically significant $P$-value. ${ }^{\text {aFactors that }}$ showed a positive association in predicting DE after HSCT in those patients without DE before HSCT. ${ }^{b}$ Factor positively associated with DE development after HSCT in those patients without DE before HSCT.

HSCT. ${ }^{17}$ The remaining studies on ocular surface involvement in HSCT only analyzed patients after HSCT. ${ }^{5,11-15,32,33}$

In our study, DE was present in 50 patients (53\%) before HSCT, this value being higher as compared with Ogawa et al ${ }^{17}$ who reported an incidence of $17 \%$ and at the upper limit of the wide range (5-50\%) reported for a general hospital-based population. ${ }^{34-39}$

The explanation could likely be related to the history of the patient population of this study, who underwent several previous chemotherapy and total body irradiation treatments before allotransplant. However, a similar prevalence was shown in a previous study from our group where a larger population of pretransplant patients had been analyzed ${ }^{20}$ and no specific previous treatments had been found to be related to DE pre-HSCT. The DE patients showed mild-to-moderate levels of severity in both signs and symptoms with a hyperevaporative DE profile. To the best of our knowledge, pre-HSCT DE profile had not been characterized previously.

After HSCT, 72 patients (77\%) were found to be affected by DE, mostly presenting tear instability and 
Table 5 Univariate odds ratios (OR) and 95\% confidence intervals of associated factors in the subgroup of patients, defined as those suffering from DE before HSCT and worsening DE after HSCT

\begin{tabular}{|c|c|c|}
\hline Variable & $\begin{array}{l}\text { Unadjusted OR } \\
\quad(95 \% \text { CI })\end{array}$ & $\mathrm{P}$ \\
\hline Recipient age (years) & $0.98(0.93-1.03)$ & 0.61 \\
\hline Recipient gender (female vs male) & $0.98(0.29-3.26)$ & 0.92 \\
\hline \multicolumn{3}{|l|}{ Ocular variables } \\
\hline CL wear & $0.69(0.21-2.24)$ & 0.53 \\
\hline VDT use & $0.25(0.15-1.08)$ & 0.28 \\
\hline Previous eye surgery & $0.31(0.18-1.23)$ & 0.38 \\
\hline \multicolumn{3}{|l|}{ Hematological variables } \\
\hline Type of disease & $0.87(0.22-3.40)$ & 0.84 \\
\hline $\begin{array}{l}\text { Time from diagnosis to HSCT } \\
\text { (>6 months) }\end{array}$ & $0.90(0.36-2.24)$ & 0.83 \\
\hline Advanced disease stage & $1.08(1.02-4.71)$ & 0.03 \\
\hline $\begin{array}{l}\text { Previous chemotherapy } \\
\text { medications (no. of cycles) }\end{array}$ & $0.94(0.79-1.13)$ & 0.55 \\
\hline \multicolumn{3}{|l|}{ Transplant variables } \\
\hline Donor-recipent sex mismatch ${ }^{\mathrm{a}}$ & $1.17(1.11-1.61)$ & 0.02 \\
\hline Donor age & $1.02(0.97-1.09)$ & 0.30 \\
\hline Donor type (related donors) ${ }^{a}$ & $1.22(1.05-2.42)$ & 0.02 \\
\hline Stem cell source $(\mathrm{PB} \text { cells })^{\mathrm{a}}$ & $1.31(1.21-1.89)$ & 0.01 \\
\hline $\begin{array}{l}\text { Intensity of conditioning } \\
\text { regimen }\end{array}$ & $0.80(0.30-2.07)$ & 0.55 \\
\hline HLA mismatch & $1.26(0.31-5.19)$ & 0.74 \\
\hline CD34+ cells infused ${ }^{a}$ & $1.03(0.99-1.04)$ & 0.76 \\
\hline GVHD systemic $^{\mathrm{b}}$ & $1.51(1.23-3.53)$ & 0.03 \\
\hline
\end{tabular}

Multiple analysis of variables significant in the univariate or clinically relevant did not show any statistically significant $P$-value. ${ }^{a}$ Factors that showed a positive association in predicting worst DE after HSCT in those patients with preexisting DE before HSCT. ${ }^{\text {b}}$ Factor positively associated with DE worsening after HSCT in those patients with DE before HSCT.

pathological MGD score, whereas the value of tear secretion as a median was found in the normal range. It is difficult to compare our results with the several previous reports in the literature only dealing with DE after HSCT, and this is due to different time interval from HSCT and ophthalmological examination, concurrent systemic therapy, underlying hematological malignancies not always specified, and inharmonious criteria for $\mathrm{DE}$ diagnosis. However, the ocular surface parameters shown after HSCT in our patients were similar to those found in mild DE populations from another study. ${ }^{40}$ In addition, MGD functional impairment found in our study is in agreement with others ${ }^{41}$ who found meibomian gland morphological alterations and loss after HSCT.

Twenty-two out of the $72 \mathrm{DE}$ patients after HSCT were classified as incident cases, that is, those not diagnosed as DE before HSCT. These patients also showed a mild-tomoderate hyperevaporative profile.

The influence of ocular, hematological, and HSCTrelated variables on the ocular GVHD or DE development
post-HSCT was investigated previously. The heterogeneity of populations studied and their treatment along with the not univocal classification for GVHD (in particular those preceding the NIH guidelines) in the previous literature make results difficult to be compared. In addition, only one study at present ${ }^{17}$ analyzed the same patients before and after HSCT, providing data both from subjects not having a DE pre-HSCT and developing a DE post-HSCT and from those worsening a DE preHSCT.

Several factors such as donor-recipient sex mismatch, increasing recipient age, and PB as stem cell source had been identified as associated factors either for ocular and systemic GVHD. 5,15,17,32,42 On the contrary, the role of the conditioning regimen, donor-recipient relation, and HLA compatibility is still unclear. $5,11,17,32,33$

In the present study, increasing recipient age and chronic systemic GVHD were confirmed as associated with DE post-HSCT. ${ }^{15,17,32}$ Recipient female gender, PB cells as stem cell source, RDs, and donor-recipient sex mismatch were found to be associated with $\mathrm{DE}$ onset post-HSCT, despite previous conflicting results from previous ophthalmological studies., $12,15,17,32$

However, some of these (increasing recipient age, PB cells as stem cell source, donor-recipient sex mismatch) are widely recognized as associated factors with GVHD onset in the hematological literature. ${ }^{42}$

Some unexpected results were found for the analysis of conditioning intensity and RDs as associated factors. The intensity of conditioning regimen was not associated with an increased risk of DE post-HSCT, in disagreement with the hematological literature ${ }^{42}$ but the great variability in protocols and treatment regimen used could have affected more reliable correlations.

RDs showed a significant association with DE postHSCT, in agreement with some authors ${ }^{11}$ but not with others. $5,12,17,42$ This finding could be attributed to the different GVHD prophylaxis given according to donor type: all unrelated transplant received ATG in addition to standard prophylaxis (Calcineurin-inhibitor+short-term Methotrexate), whereas only roughly half patients received ATG in the HLA-identical sibling setting. As recently reported, ATG reduces the incidence and severity of cGVHD after allogeneic transplant from HLA-identical sibling PB stem cells; in particular, ocular GVHD appear to be dramatically reduced on the arm with ATG. ${ }^{43}$

We have also analyzed potential risk factors not previously evaluated in the ophthalmological literature; of these, time interval from diagnosis to HSCT and donor age were not found associated with DE after HSCT, whereas advanced stage of hematological malignancy and the number of stem cells infused were found associated with DE after HSCT. 
In our series, the severity of post-HSCT ocular surface impairment was not as high as reported by other authors. ${ }^{5,11-15}$ No corneal complication occurred unlike results from Tabbara et $a l^{6}$ in which half GVHD patients suffered from corneal ulcers. Concordantly, no patient developed DE post-HSCT so severe to be classified as DEWS worst severity score 4 . A possible explanation is that an ocular therapy had been administered to all those patients diagnosed as DE before HSCT, already before starting the conditioning regimen, and this could have tempered a DE progression after transplant. This hypothesis appears in agreement with others ${ }^{44}$ who suggested the effectiveness of pre-HSCT initiation of therapy before HSCT for the treatment and prophylaxis of DE after HSCT.

According to NIH GVHD criteria, ocular GVHD is diagnosed in the event of a new onset of dry, gritty, painful eyes, cicatricial conjunctivitis, keratoconjunctivitis sicca, or confluent areas of punctate keratopathy observed after HSCT. Ocular involvement represents a distinctive sign and therefore not considered to be sufficient as alone to establish an unequivocal diagnosis for general chronic GVHD trials. ${ }^{10}$ Following these guidelines, systemic chronic GVHD was diagnosed in $42 \%$ of our patients as a whole, whereas ocular chronic GVHD in $35.5 \%$, regardless the pre-HSCT ocular impairment. As a matter of fact, if a patient already suffers from DE disease before HSCT, ocular surface impairment evaluated at the postHSCT check-up cannot be considered as 'incident cases' and cannot be diagnosed as post-HSCT ocular GVHD. This finding influenced the real prevalence of ocular GVHD after HSCT, which decreased in the present study to $12 \%$.

As already reported, a poor diagnostic performance in diagnosing DE already before HSCT was found for the Schirmer test, which has been recently removed in the NIH Guidelines from the markers of severity and from the response criteria. ${ }^{10,45}$ In addition, as the Schirmer score does not reflect changes in ocular GVHD activity, it was not recommended for the measurement of the changes in ocular GVHD studies by the Chronic GVHD Consortium. ${ }^{46}$

We recognize that in this study more variables potentially needed to elucidate the onset and development of ocular GVHD were lacking as not found in our records. This limitation occurs in any retrospective study based on chart reviews; however, this is a major initial study generating data to be verified further by larger cohort prospective studies.

In conclusion, this study showed that DE is already present before HSCT. This finding demonstrates that comprehensive pretransplant assessment of ocular surface is highly recommended, as it has been already recognized for functional respiratory values in lung GVHD. ${ }^{10,45}$ This recommendation is not only addressed to an accurate early diagnosis but also to a prompt treatment with a direct influence on the ocular GVHD onset and severity.

Further larger prospective multicenter studies based on pretransplantation and posttransplantation ophthalmic evaluation are needed to identify overall associated factors and the actual prevalence of ocular GVHD after HSCT.

\section{Summary}

What was known before

- Several studies described ocular surface impairment and GVHD onset in hematological patients after HSCT. On the contrary, little information is available about ocular surface changes after HSCT compared with pretransplant baseline condition.

What this study adds

- Dry eye (DE) is already present before HSCT, and this is the main risk factor for DE development after HSCT. Data support the recent recommendation in that ophthalmological evaluation should be included in the protocol before the HSCT procedure, to identify potential risk factors, treat DE patients early and reduce post-HSCT damages.

\section{Conflict of interest}

The authors declare no conflict of interest.

\section{Acknowledgements}

We thank the Epidemiology and Biostatistics Unit, Research and Innovation Department Sant'OrsolaMalpighi Hospital and the University of Bologna for the support in the statistical analyses of data.

\section{References}

1 Arai S, Jagasia M, Storer B, Chai X, Pidala J, Cutler C et al. Global and organ-specific chronic graft-versus-host disease severity according to the 2005 NIH Consensus Criteria. Blood 2011; 118: 4242-4249.

2 Pidala J, Anasetti C, Jim H. Quality of life after allogenic hematopoietic cell transplantation. Blood 2009; 114: 7-19.

3 Kim SK. Ocular graft vs. host disease. Ocul Surf 2005; 3: S177-S179.

4 Nassiri N, Eslani M, Panahi N, Mehravaran S, Ziaei A, Djalilian AR. Ocular graft versus host disease following allogeneic stem cell transplantation: a review of current knowledge and recommendations. J Ophthalmic Vis Res 2013; 8: $351-358$

5 Jacobs R, Tran U, Chen H, Kassim A, Engelhardt BG, Greer JP et al. Prevalence and risk factors associated with development of ocular GVHD defined by NIH consensus criteria. Bone Marrow Transplant 2012; 47: 1470-1473. 
6 Tabbara KF, Al-Ghamdi A, Al-Mohareb F, Ayas M, Chaudhri N, Al-Sharif F et al. Ocular findings after allogenic hematopoietic stem cell transplantation. Ophthalmology 2009; 116: $1624-1629$.

7 Filipovich AH, Weisdorf D, Pavletic S, Socie G, Wingard JR, Lee SJ et al. National Institutes of Health Consensus Development Project on Criteria for Clinical Trials in Chronic Graft-versus-Host Disease: I. Diagnosis and Staging Working Group Report. Biol Blood Marrow Transplant 2005; 11: 945-955.

8 Hessen M, Akpek EK. Ocular graft-versus-host disease. Curr Opin Allergy Clin Immunol 2012; 12: 540-547.

9 Kim SK. Update on ocular graft versus host disease. Curr Opin Ophthalmol 2006; 17: 344-348.

10 Jagasia MH, Greinix HT, Arora M, Williams KM, Wolff D, Cowen EW et al. National Institutes of Health Consensus Development Project on Criteria for Clinical Trials in Chronic Graft-versus-Host Disease: I. The 2014 Diagnosis and Staging Working Group Report. Biol Blood Marrow Transplant 2015; 21: 389-401.

11 Westeneng AC, Hettinga Y, Lokhorst H, Verdonck L, van Dorp S, Rothova A. Ocular graft-versus-host disease after allogenic stem cell transplantation. Cornea 2010; 29: 758-763.

12 Shikari H, Amparo F, Saboo U, Dana R. Onset of ocular graft-versus-host disease symptoms after allogenic hematopoietic stem cell transplantation. Cornea 2015; 34: 243-247.

13 Ivanir Y, Shimoni A, Ezra-Nimni O, Barequet IS. Prevalence of dry eye syndrome after allogenic hematopoietic stem cell transplantation. Cornea 2013; 32: e97-e101.

14 Vanathi M, Kashyap S, Khan R, Seth T, Mishra P, Mahapatra $\mathrm{M}$ et al. Ocular surface evaluation in allogenic hematopoietic stem cell transplantation patients. Eur J Ophthalmol 2014; 24: 655-666.

15 Tichelli A, Duell T, Weiss M, Socie G, Ljungman P, Cohen A et al. Late-onset keratoconjunctivitis sicca syndrome after bone marrow transplantation: incidence and risk factors. European Group or Blood and Marrow Transplantation (EBMT) Working Party on late effects. Bone Marrow Transplant 1996; 17: 1105-1111.

16 Przepiorka D, Anderlini P, Saliba R, Cleary K, Mehra R, Khouri I et al. Chronic graft-versus-host disease after allogenic blood stem cell transplantation. Blood 2001; 98: 1695-1700.

17 Ogawa Y, Okamoto S, Wakui M, Watanabe R, Yamada M, Yoshino $\mathrm{M}$ et al. Dry eye after haematopoietic stem cell transplantation. Br J Ophthalmol 1999; 83: 1125-1130.

18 Ogawa Y, Kim SK, Dana R, Clayton J, Jain S, Rosenblatt MI et al. International Chronic Ocular Graft-vs-Host Disease (GVHD) Consensus Group: proposed criteria for chronic GVHD (Part I). Sci Rep 2013; 3: 3419.

19 Dietrich-Ntoukas T, Cursiefen C, Westekemper H, Eberwein P, Reinhard T, Bertz $\mathrm{H}$ et al. Diagnosis and treatment of ocular chronic graft-versus-host disease: Report from the German-Austrian-Swiss Consensus Conference on Clinical Practice in Chronic GVHD. Cornea 2012; 31: 299-310.

20 Giannaccare G, Bonifazi F, Sessa M, Fresina M, Arpinati M, Bandini G et al. Dry eye disease is already present in hematological patients before hematopoietic stem cell transplantation. Cornea 2016; 35: 638-643.

21 Schiffman RM, Christanson MD, Jacobsen G, Hirsch JD Reis BL. Reliability and validity of the Ocular Surface Disease Index. Arch Ophthalmol 2000; 118: 615-621.
22 Lemp MA. Report of the National Eye Institute/Industry Workshop on clinical trials in dry eye. CLAO J 1995; 21: 221-232.

23 Van Bijsterveld OP. Diagnostic tests in the sicca syndrome. Arch Ophthalmol 1969; 82: 10-14.

24 Jabs DA, Wingard J, Green WR, Farmer ER, Vogelsang G, Saral R. The eye in bone marrow transplantation. III. Conjunctival graft-vs-host disease. Arch Ophthalmol 1989; 107: 1343-1348.

25 Foulks GN, Bron AJ. Meibomian gland dysfunction: a clinical scheme for description, diagnosis, classification, and grading. Ocul Surf 2003; 1: 107-126.

26 The epidemiology of dry eye disease: Report of the Epidemiology Subcommittee of the International Dry Eye WorkShop. (2007). Ocul Surf 2007; 5: 93-107.

27 Sullivan BD, Whitmer D, Nichols KK, Tomlinson A, Foulks GN, Geerling G et al. An objective approach to dry eye disease severity. Invest Ophthalmol Vis Sci 2010; 51: 6125-6130.

28 Pflugfelder SC, Maskin SL, Anderson B et al. A randomized, doublemasked, placebo-controlled, multicenter comparison of loteprednol etabonate ophthalmic suspension, $0.5 \%$, and placebo for treatment of keratoconjunctivitis sicca in patients with delayed tear clearance. Am J Ophthalmol 2004; 138: $444-457$.

29 Wan KH, Chen LJ, Young AL. Efficacy and safety of topical $0.05 \%$ cyclosporine eye drops in the treatment of dry eye syndrome: a systematic review and meta-analysis. Ocul Surf 2015; 13: 213-225.

30 Management and therapy of dry eye disease: Report of the Management and Therapy Subcommittee of the International Dry Eye WorkShop (2007). Ocul Surf 2007; 5: 163-178.

31 Schaumberg DA, Sullivan DA, Dana MR. Epidemiology of dry eye syndrome. Adv Exp Med Biol 2002; 506: 989-998.

32 Leite SC, de Castro RS, Alves M, Cunha DA, Correa ME, de Silveira LA et al. Risk factors and characteristics of ocular complications, and efficacy of autologous serum tears after hematopoietic stem cell transplantation. Bone Marrow Transplant 2006; 38: 223-227.

33 Wang JC, Teichman JC, Mustafa M, O'Donnell H, Broady R, Yeung SN. Risk factors for the development of ocular graftversus-host disease (GVHD) dry eye syndrome in patients with chronic GVHD. Br J Ophthalmol 2015; 99: 1514-1518.

34 Li J, Zheng K, Deng Z, Zheng J, Ma H, Sun L et al. Prevalence and risk factors of dry eye disease among a hospital-based population in southeast China. Eye Contact Lens 2015; 41: 44-50.

35 Hikichi T, Yoshida A, Fukui Y, Hamano T, Ri M, Araki K et al. Prevalence of dry eye in Japanese eye centers. Graefes Arch Clin Exp Ophthalmol 1995; 233: 555-558.

36 Onwubiko SN, Eze BI, Udeh NN, Arinze OC, Onwasigwe EN, Umeh RE. Dry eye disease: prevalence, distribution and determinants in a hospital-based population. Cont Lens Anterior Eye 2014; 37: 157-161.

37 Basak SK, Pal PP, Basak S, Bandyopadhyay A, Choudhury S, Sar S. Prevalence of dry eye diseases in hospital-based population in West Bengal, Eastern India. J Indian Med Assoc 2012; 110: 789-794.

38 Sahai A, Malik P. Dry eye: prevalence and attributable risk factors in a hospital-based population. Indian J Ophthalmol 2005; 53: 87-91. 
39 Shah S, Jani H. Prevalence and associated factors of dry eye: our experience in patients above 40 years of age at a Tertiary Care Center. Oman J Ophthalmol 2015; 8: 151-156.

40 Wang Y, Ogawa Y, Dogru M, Tatematsu Y, Uchino M, Kamoi $\mathrm{M}$ et al. Baseline profiles of ocular surface and tear dynamics after allogeneic hematopoietic stem celltransplantation in patients with or without chronic GVHD-related dry eye. Bone Marrow Transplant 2010; 45: 1077-1083.

41 Engel LA, S Wittig S, Bock F, Sauerbier L, Scheid C, Holtick $U$ et al. Meibography and meibomian gland measurements in ocular graft-versus-host disease. Bone Marrow Transplant 2015; 50: 961-967.

42 Socié G, Ritz J. Current issues in chronic graft-versus-host disease. Blood 2014; 124: 374-384.

43 Kroger N, Solano C, Wolschke C, Bandini G, Patriarca F, Pini $\mathrm{M}$ et al. Antilymphocyte globuline for prevention of chronic graft-versus-host disease. N Engl J Med 2016; 374: 43-53.

44 Boynton GE, Raoof D, Niziol LM, Hussain M, Mian SI. Prospective randomized trial comparing efficacy of topical loteprednol etabonate $0.5 \%$ versus cyclosporine-A $0.05 \%$ for Treatment of dry eye syndrome following hematopoietic stem cell transplantation. Cornea 2015; 34: 725-732.

45 Lee SJ, Wolff D, Kitko C, Koreth J, Inamoto Y, Jagasia M et al. Measuring therapeutic response in chronic graft-versus-host disease. National Institutes of Health consensus development project on criteria for clinical trials in chronic graft-versus-host disease: IV. The 2014 Response Criteria Working Group report. Biol Blood Marrow Transplant 2015; 21: 984-999.

46 Inamoto Y, Chai X, Kurland BF, Cutler C, Flowers ME, Palmer JM et al. Validation of measurement scales in ocular graft-versus-host disease. Ophthalmology 2012; 119: 487-493. 